\title{
Triplo tabu: sobre o suicídio na infância e na adolescência
}

\author{
Triple taboo: considerations about suicide among children \\ and adolescents
}

Orli Carvalho da Silva Filho (https://orcid.org/0000-0002-5268-6097) ${ }^{1}$

Maria Cecília de Souza Minayo (https://orcid.org/0000-0001-6187-9301) ${ }^{2}$

\footnotetext{
${ }^{1}$ Instituto Nacional da Saúde da Mulher, da Criança e do Adolescente Fernandes Figueira, Fundação Oswaldo Cruz (Fiocruz). Av. Rui Barbosa 716, Flamengo. 22250-020 Rio de Janeiro RJ Brasil. orli.filho@iff.fiocruz.br ${ }^{2}$ Departamento Estudos sobre Violência e Saúde Jorge Careli, Escola Nacional de Saúde Pública Sergio Arouca, Fiocruz. Rio de Janeiro RJ Brasil.
}

\begin{abstract}
This article aims to understand the construction and repercussion of taboos involving suicides among children and adolescents, considering the discomfort, silence, and dread that the theme causes across society. Due to the recognition of a continuum of taboos (taboo of death $<$ taboo of suicide < taboo of child suicide), the authors present, as an attempt to address this issue, the concept of a triple taboo, recognizing the incommensurability of self-inflicted deaths that have children and adolescents as protagonists. Developed from a qualitative study with paediatricians during their medical residence, this paper serves as a call to professionals who assist children and adolescents in the country. Paediatric training needs to recognise these taboos and the dimensions of suicidal behaviour as a manifestation of violence and as a threat to mental health. Their identification is a critical and urgent element in contemporary children and adolescents' care.
\end{abstract}

Key words Death, Suicide, Taboo, Child, Adolescent
Resumo Este artigo pretende compreender a construção e a repercussão dos tabus que envolvem o suicídio de crianças e adolescentes, considerando o incômodo, o silêncio e o pavor que esse tema provoca em toda a sociedade. Diante do reconhecimento de um continuum de tabus (tabu da morte $<$ tabu do suicídio < tabu do suicídio infantojuvenil), propôs-se, como tentativa de clarificar essa questão, o conceito de um triplo tabu, valorizando a incomensurabilidade das mortes autoprovocadas que têm as crianças e os adolescentes como protagonistas. Tendo sido desenvolvido a partir de um estudo qualitativo com pediatras em formação, este documento se configura como um chamado aos profissionais que assistem crianças e adolescentes no país. É preciso que a formação pediátrica reconheça esses tabus e as dimensões do comportamento suicida como uma manifestação de violência e um agravo à saúde mental, identificando-o como um elemento crítico e urgente na assistência contemporânea a crianças e adolescentes.

Palavras-chave Morte, Suicídio, Tabu, Criança, Adolescente 


\section{Introdução}

O objetivo deste artigo é apresentar o conceito de triplo tabu que permeia o fenômeno do suicídio de crianças e adolescentes no Brasil. Sua proposição se deu a partir de ideias preconcebidas e em continuum que se somam e se sobrepõem, ainda que parcialmente discriminadas, sobre o comportamento suicida: tabu da morte $<$ tabu do suicídio $<$ tabu do suicídio infantojuvenil. Esse último é aqui compreendido e apontado como um tabu maior, que incorpora os seus dois precedentes - um triplo tabu -, dada a incomensurabilidade do suicídio quando seu protagonista é uma criança ou um adolescente.

A ideia de um triplo tabu foi desenvolvida pelos autores deste artigo no estudo "Percepção e conhecimento de médicos residentes em pediatria no Rio de Janeiro sobre comportamento suicida na infância e adolescência", aprovado pelo Comitê de Ética em Pesquisa do Instituto Nacional da Saúde da Mulher, da Criança e do Adolescente Fernandes Figueira/Fundação Oswaldo Cruz $^{1}$. A pesquisa realizada, entre 2018 e 2019, com pediatras em formação demonstrou lacunas no conhecimento desse fenômeno, assim como a construção e a propagação de (pré)conceitos que distanciam os profissionais dessa temática, dificultando a abordagem de crianças e adolescentes em sofrimento psíquico e cristalizando as ideias preconcebidas presentes na sociedade ${ }^{2}$.

É importante esclarecer que numa sociedade ocidental e de formação cristã como a brasileira, a morte autoinflingida sempre foi considerada um objeto de repúdio por desvios religiosos, culturais e legais ${ }^{3}$. Cada vez mais, porém, estudos sociológicos, psicológicos e psiquiátricos mostram sua ocorrência como um fato plausível desde a infância, associado a sofrimento emocional, agravos de saúde e questões macro e microssociais $^{4-9}$. No entanto, a tendência das famílias, das instituições e das comunidades é a sua interdição, revelando uma extrema dificuldade e preconceito em lidar com esse fenômeno, ou sua categorização como um problema de exclusiva causalidade biomédica. Se por um lado há um grande alarde, amplificado pela mídia em seus novos formatos $^{8,10,11}$, sobre os riscos de autoagressões por esse grupo etário, por outro, prevalecem a inabilidade e o silêncio para abordá-las².

Apesar do silêncio social e do distanciamento profissional sobre o suicídio de crianças e adolescentes, o cenário clínico-epidemiológico pediátrico atual no Brasil e no mundo encontra-se fortemente marcado pela morbimortalidade por causas externas e pela crescente prevalência de transtornos mentais ${ }^{12-14}$. Dados nacionais corroboram a tendência de crescimento na prevalência de ideação suicida e na incidência de suicídio na população de 10 a 19 anos, principalmente se considerado o subgrupo de 15 a 19 anos $^{15-17}$. Tendo como referência o diverso universo de adolescentes e jovens de 15 a 29 anos, tem-se o suicídio como uma das suas principais causas de morte $^{17,18}$. O comportamento suicida assim, enquanto manifestação de violência e de um evidente agravo emocional ${ }^{4,5,19}$, precisa se tornar um tópico prioritário do cuidado integral a crianças e adolescentes, constituindo formalmente parte da agenda das áreas da saúde, da educação e da assistência social. Dessa forma, reconhece-se a necessidade de um maior esclarecimento e problematização desse tema, identificando elementos que possam contribuir para a sua adequada compreensão e manejo.

A partir de elementos desenvolvidos na pesquisa supracitada ${ }^{1}$, serão aqui tratados alguns apontamentos sobre o continuum de tabus que circunscrevem esse fenômeno: o tabu da morte, o tabu do suicídio e o tabu do suicídio infantojuvenil. Reconheceu-se, nesta pesquisa, a relação do conceito de tabu com a ideia do proibido; ou seja, a noção de uma proibição, presente e válida em determinada cultura, de se mencionar, tocar ou se aproximar daquilo que é entendido como perigoso, impuro, misterioso ou inexplicável ${ }^{20,21}$.

\section{Tabu da morte}

Apesar da notória aceitação do suicídio como uma espécie de morte, ressalta-se esse ponto para a condução compreensiva do espectro de tabus, a partir do qual não é possível se chegar ao suicídio sem antes se passar pelo confronto com a morte. De modo que os suicídios carregam em si os estigmas, os receios e as fantasias da morte.

Saber e até prever o próprio fim, importante distinção da espécie humana frente aos outros seres viventes na Terra, constituí uma peculiaridade fundante do homem e sua existência, tendo impulsionado diferentes formas de lidar com a terminalidade, a morte, os moribundos e os $\operatorname{mortos}^{20,22}$. Ainda que a morte seja um evento natural e biológico constituinte de todo o ciclo vital, o processo do morrer deve ser compreendido social e culturalmente, inscrito no tempo e no espaço; nesse panorama, sugere Elias ${ }^{22}$ que "a morte é um problema dos vivos. Os mortos não têm problemas."22(p.10).

Apresentando as transformações deflagradas pelo processo civilizador que levaram à individualização e o silêncio do senso coletivo sobre a 
morte, Menezes ${ }^{23}$ aponta, em referência a Norbert Elias:

Há várias formas de os indivíduos lidarem com a ideia da finitude da vida: pode-se evitar a ideia da morte através da mitologização do final da vida, do encobrimento da ideia indesejada, pela crença na própria imortalidade ou encará-la como um fato da existência e ajustar a vida diante dessa realidade. Para Elias, atualmente há uma tendência à crença na imortalidade e ao afastamento da ideia da morte. ${ }^{23}$ (p.147).

Tal recalque sobre a morte, ao longo das gerações, esvaziou os rituais seculares de sentimento e de significado, estimulando a autonomia e a individualidade para esse lidar, dando espaços a lacunas e vazios semânticos, propiciando o surgimento de silêncios e tabus ${ }^{20,22}$.

O mesmo autor avança em sua argumentação provocando que esse tabu trava a língua e as mãos da sociedade, impedindo a demonstração de afetos e desautorizando os rituais coletivos, aumentando a distância dos vivos para com seus mortos e moribundos ${ }^{22}$. Justificado pelos avanços médicos e científicos, fomentando essa distância, o cenário da morte deslocou-se para os hospitais, higienizando-a e terceirizando- $\mathrm{a}^{24}$. Uma morte asséptica, silenciosa, isolada, "uma área vazia no mapa social”22(p.36): um tabu.

Assim, o silêncio sobre a morte é reforçado por seu deslocamento para os hospitais, sede, símbolo e polo formador da prática médica, de modo que esse tabu repercute e cristaliza. Há progressos para o enfrentamento da morte, mas não para o diálogo com ela, interditando-a do cotidiano $^{25}$.

Um contraponto, porém, precisa ser considerado quando a formação social e cultural brasileira está na agenda: o tabu da morte e a concomitância com a banalização para com algumas mortes. E aqui se destacam as mortes violentas e precoces de minorias étnicas e raciais ${ }^{26}$; não por tais eventos serem representativos de uma ruptura ou reconstrução desse tabu, mas por ainda concretizarem uma perpetuação discriminatória do processo colonialista. A vulgarização desses óbitos, contrariamente ao que pode inicialmente sugerir, imputa ainda mais o silêncio segregador e moralizador, não sendo capaz de provocar questionamentos sobre a lancinante finalização da vida, pelo fato do menor valor que essas vidas parecem ter.

O constrangimento no lidar com a consciência do aniquilamento individual e coletivo é imbuído de traços fundamentais de socialização e cultura ${ }^{23}$. De forma que, a identificação e o re- conhecimento do tabu da morte não representam um juízo em si, mas um dispositivo para a compreensão do senso comum de como a morte, em seus diferentes formatos e por seus diferentes agentes, apresenta-se na contemporaneidade.

\section{Tabu do suicídio}

Para além das tentativas de explicações acadêmicas sobre a morte autoprovocada, como o mais grave desfecho do comportamento suicida associado a transtornos mentais ${ }^{19}$ ou ainda como um fato social entendido e modulado a partir das dimensões durkheimianas de integração e regulação ${ }^{27}$, ousa-se propor que sua descrição como um fenômeno autoinflingido seja a marca que mais desperta complexidade e incômodo. O suicídio, geralmente, é qualificado como uma categoria de morte diferente das demais. Ainda que em outros eventos violentos fatais também se constate uma brusca antecipação no curso natural da vida em sua diária aproximação com a morte, a intencionalidade (ainda que teórica) ${ }^{4}$ do suicídio confere uma transgressão contra a sobrevivência humana, representando uma dupla agressão à humanidade.

O constrangimento com a morte é assim ampliado num cenário onde o mesmo personagem é vítima e culpado, ferindo o espaço social numa reapresentação do silêncio e do tabu - duplo tabu - conforme proposto por Dias ${ }^{28}$.

Desta forma, podemos supor que o tabu imposto ao falar da morte repercute sobre o suicida, impedindo-o de se comunicar abertamente sobre seus motivos - o que, de um lado, impossibilita a ajuda social na superação de seus impasses, se for o caso, $e$ de outro lado, contribui para a constituição de um grande enigma em torno do tema. ${ }^{28}$ (p.38).

Como descreve Dias ${ }^{28}$, o silêncio da morte se estende ao suicídio e ao suicida; de uma possibilidade polifônica e polissêmica tem-se uma imposição paradigmática do não dizer ${ }^{3}$. Nessa interdição, é possível identificar a alocação do suicídio na categoria de desvio, o que corrobora sua concepção como um tabu. Três modelos na história do Ocidente prevalecem e se sobrepõem nesse entendimento do suicídio como desvio: pecado, crime e doença ${ }^{3}$. Ainda que tal discussão extrapole o escopo desse artigo, destaca-se que a tonalidade desviante que historicamente se conferiu ao suicídio tem fomentado diversos elementos de exclusão, aprisionando-o ainda mais aos constrangimentos sociais das mortes.

Não é novo apontar o tabu do suicídio, num momento que até mesmo campanhas de preven- 
ção e materiais educativos disponibilizados em diferentes mídias já se debruçaram sobre isso ${ }^{4,5}$. A construção do espectro de tabus aqui defendida baseia-se na clareza de que não é possível avançar em reflexões sobre o comportamento suicida - e o suicídio em especial - se não for possível o diálogo com a morte e com os moribundos. Como já sugerido nesse corpus, a presença e o protagonismo infantojuvenis nessa e sobre essa morte singular, porém universal, conferem silêncios e tabus mais constrangedores: um triplo tabu.

\section{Triplo tabu}

Mesmo se apoiando na lógica de que o suicídio é definido como um desfecho do comportamento suicida ${ }^{5,7}$, não é difícil imaginar que também pode representar alguns começos, como o do sofrimento dos sobreviventes e enlutados e o do lidar com o seu tabu. A singularidade representada por essa morte exige dos vivos que perderam um ente por suicídio um recomeço mais doloroso. Carregam as marcas familiares ou afetivas de um suicida e, por isso, podem carecer de cuidados e estratégias de prevenção, devido ao impacto que essa morte pode ter em suas vidas ${ }^{4}$. Nesse espectro, a interrupção das vidas infantis bondosas, angelicais e que simbolizam a permanência da espécie - provoca um estranhamento adicional, ferindo a expectativa que a humanidade desenvolveu para além do cuidado instintivo com suas proles.

O deslocamento da morte dos espaços cotidianos no decorrer do processo civilizador ${ }^{22}$ veio acompanhado de um elemento pediátrico: a valorização dos cuidados com a saúde das crianças e a aposta nelas como uma esperança do porvir ${ }^{29}$. Pode-se considerar que o nascimento da Pediatria enquanto especialidade médica se vincula histórica e socialmente à comoção e ao combate à mortalidade infantil. Numa reflexão sobre o surgimento da Pediatria brasileira, Pereira afirma ${ }^{29}$ :

Os nascentes pediatras reclamariam para si o papel de protetores da vida, aqueles capazes de livrar as famílias do estigma da mortalidade e, sobretudo, consentâneos do ideal de condução da prole a um suposto destino de saúde, robustez e vitalidade. ${ }^{29}$ (p.15).

Justifica-se, desse modo, um ethos pediátrico, aqui replicado aos demais profissionais e atores sociais que lidam com o público infantil, investido no combate à mortalidade, à imoralidade $\mathrm{e}$ aos padrões desviantes que ameaçam as crianças e os adolescentes. Um ethos que, ainda que auto- rizado socialmente a assumir a voz dos "infantes", não consegue conviver com os seus óbitos, nem dialogar com suas situações de finitude e de limitação de cuidados ou ainda estimulá-los a uma franca reflexão sobre a morte ${ }^{2,29}$.

Instrumentalizada por essa firme perspectiva de defesa e proteção da vida infantojuvenil, uma das responsáveis pelo aumento da sobrevida desse grupo etário ${ }^{12,14}$, compreende-se o silêncio sobre as mortes pediátricas e a incomensurabilidade sobre a sua condição autoprovocada. Firmeza, porém, cujo constrangimento tem impedido novas e efetivas estratégias de enfrentamento da morbidade e da mortalidade nesse grupo etário. Desconsiderar e não saber lidar com a realidade do comportamento suicida infantojuvenil têm se revelado um importante entrave clínico e epidemiológico para a assistência pediátrica na sociedade brasileira, atitudes que denunciam a penetrância e a solidez do triplo tabu na formação profissional. Um espaço e um contexto de formação pediátrica que silenciados e contidos nesses e por três tabus mostram-se menos hábeis para cumprir o propósito que os sustentam, travando e limitando planos e ações de promoção, prevenção, tratamento e reabilitação.

Acrescenta-se que a representação meiga, dócil e angelical das crianças no imaginário social e dos pediatras distancia-os das temáticas existenciais que povoam o cotidiano assistencial ainda que diante de desfechos potencialmente fatais ${ }^{29}$. Dessa forma, a morte ou a lesão autoinfligidas de uma criança ou de um adolescente geram um tabu e um incômodo ainda maiores, dificultando intervenções possíveis. Como um dos grupos autorizados a esse cuidado e controle, a percepção desses profissionais é normativa dos valores transmitidos às famílias e a grupos sociais ${ }^{29}$ que assumem a inadmissibilidade do comportamento suicida em crianças e adolescentes mesmo que suas próprias vivências e o conhecimento epidemiológico reflitam outro cenário.

Concebendo ser o suicídio a morte que mais problematiza a finitude humana presente também na existência de crianças e adolescentes, fornecem-se as premissas para a compreensão do triplo tabu aqui apresentado. É reconhecido que a produção de categorias pode proporcionar uma limitação e rigidez ao conhecimento, ainda assim, faz-se aqui uma tentativa inversa: ampliar as fronteiras de interpretação sobre o fenômeno do suicídio; não o circunscrevendo, mas o reconhecendo em suas plurais dimensões ${ }^{3}$. 


\section{Considerações finais}

Não se pretendeu, nesse artigo, insinuar os graus de intensidade de sofrimento quantificando ou ranqueando a dor de enlutados e sobreviventes dos óbitos, sejam esses autoprovocados ou não. O objetivo foi tentar compreender a construção e a repercussão desses tabus. Tabus que têm travado diálogos abertos sobre o tema e, por isso, impedido a difusão de ações de cuidado e prevenção do comportamento suicida e do suicídio de crianças e adolescentes que, conforme define Shneidman (apud Saraiva ${ }^{30}$ ), congregam um máximo de dor, perturbação e pressão.

Finaliza-se, desse modo, com uma orientação aos profissionais que assistem crianças e adolescentes no país, em especial os que atuam como formadores da área da saúde e da educação. Os silêncios e os tabus acerca da morte, do suicídio e do suicídio infantojuvenil não estão apenas presentes, mas arraigados nos cenários e práticas pediátricas. A transição epidemiológica experimentada pela pediatria brasileira e a definição de uma "Nova Pediatria"12 não foram suficientes para uma apropriação crítica de conceitos e debates que se apresentam na prática clínica com esse público, proporcionando pavor e incômodo aos profissionais. Lacunas formativas podem ser identificadas, reproduzindo nos cenários de ensino e aprendizagem o senso comum, impedindo a modulação de um espaço que intervenha sobre uma realidade clínica e epidemiológica evidente e preocupante, que perpassa a violência e o sofrimento psíquico ${ }^{1}$.

É preciso mais do que o reconhecimento do que esse texto nomeou como triplo tabu, mas o desenvolvimento de estratégias, argumentos e dispositivos para diálogos sensíveis sobre a morte e o suicídio. É necessário também reconhecer as vivências e as linguagens pediátricas, escutando e autorizando, sem as amarras dos conflitos geracionais, a voz e a subjetividade de crianças e adolescentes em suas plurais expressões, manifestações, formatos e conflitos, sobretudo quando falam do seu sofrimento.

\section{Colaboradores}

OC Silva Filho e MCS Minayo participaram da concepção e execução do estudo, assim como da elaboração, revisão e aprovação da versão final desse manuscrito. 


\section{Referências}

1. Silva Filho OC. Percepção e conhecimento de médicos residentes em pediatria no Rio de janeiro sobre comportamento suicida na infância e na adolescência [dissertação]. Rio de Janeiro: Instituto Nacional da Criança, da Mulher e do Adolescente Fernandes Figueira, Fundação Oswaldo Cruz; 2019.

2. Silva Filho OC, Minayo MCS. Comportamento suicida em adolescentes: desafios e reflexões para os pediatras brasileiros. Adolesc Saude 2018; 15(Supl.):68-72.

3. Lopes FH. Suicídio \& saber médico: estratégias históricas de dominio, controle e intervenção no Brasil do século XIX. Rio de Janeiro: Apicuri; 2008.

4. World Health Organization (WHO). Preventing suicide: a global imperative [Internet]. Geneva: WHO; 2014 [acessado 2020 fev 04]. Disponível em: http:// www.who.int/mental_health/suicide-prevention/ world_report_2014/en/.

5. Jans T, Vloet TD, Taneli Y, Warnke A. Suicide and self-harming behaviour. In: Rey JM, editor. IACAPAP E-textb Child Adolescent Mental Health. Geneva: IACAPAP; 2018.

6. Sousa GS, Santos MSP, Silva ATP, Perrelli JGA, Sougey EB. Revisão de literatura sobre suicídio na infância. Cien Saude Colet 2017; 22(9):3099-3110.

7. Hawton K, Saunders KEA, O'Connor RC. Self-harm and suicide in adolescents. Lancet 2012; 379(9834):2373-2382.

8. Niederkrotenthaler T, Stack S, Till B, Sinyor M, Pirkis J, Garcia D, Rockett IRH, Tran US. Association of Increased Youth Suicides in the United States with the Release of 13 Reasons Why. JAMA Psychiatry 2019; 76(9):933-940.

9. Dervic K, Oquendo MA. Suicidal and Self-harming Preschoolers. J Am Acad Child Adolesc Psychiatry 2019; 58(1):22-24.

10. Ortiz P, Khin EK. Traditional and new media's influence on suicidal behavior and contagion. Behav Sci Law 2018; 36(2):245-256.

11. Sousa DF, Quirino Filho JD, Cavalcanti RCPB, Santos $\mathrm{AB}$, Rolim Neto ML. The impact of the 'Blue Whale' game in the rates of suicide: Short psychological analysis of the phenomenon. Int J Soc Psychiatry 2017; 63(8):796-797.

12. Moreira MEL, Goldani MZ. A criança é o pai do homem: novos desafios para a área de saúde da criança. Cien Saude Colet 2010; 15(2):321-327.

13. Assis SG, Avanci JQ, Pesce RP, Ximenes LF. Situação de crianças e adolescentes brasileiros em relação à saúde mental e violência. Cien Saude Colet 2009; 14(2):349-361.

14. Victora CG, Aquino EM, Leal MC, Monteiro CA, Barros FC, Szwarcwald CL. Maternal and child health in Brazil: Progress and challenges. Lancet 2011; 377(9780):1868-1876.

15. Soares FC, Hardman CM, Rangel Junior JFB, Bezerra J, Petribú K, Mota J, Barros MVG, Lima RA. Secular trends in suicidal ideation and associated factors among adolescents. Braz J Psychiatry 2020; 42(5):475480 .
16. Jaen-Varas DC, Mari JJ, Asevedo E, Borschmann R, Diniz E, Ziebold C, Gadelha A. Estudo ecológico de 10 anos sobre os métodos de suicídio usados por adolescentes brasileiros. Cad Saude Publica 2020; 36(8): e00104619.

17. Brasil. Ministério da Saúde (MS). Secretaria de vigilância em Saúde. Boletim Epidemiológico vol 50. № 24. Brasília: MS; 2019.

18. Jaen-Varas D, Mari JJ, Asevedo E, Borschmann R, Diniz E, Ziebold C, Gadelha A. The association between adolescent suicide rates and socioeconomic indicators in Brazil: a 10-year retrospective ecological study. Braz J Psychiatry 2019; 41(5):389-395.

19. Bertolote JM, Fleischmann A. Suicide and psychiatric diagnosis: a worldwide perspective. World Psychiatry 2002; 1(3):181-185.

20. Pereira JC. Procedimentos para lidar com o tabu da morte. Cien Saude Colet 2013; 18(9):2699-2709.

21. Patricio N, Baudin C, Celio SN. Quando o trabalho real é tabu: introdução. Laboreal 2020; 16(1):1-8.

22. Elias N. A Solidão dos Moribundos. Rio de Janeiro: Zahar; 2001.

23. Menezes RA. A solidão dos moribundos: falando abertamente sobre a morte. Physis Rev Saude Colet 2004; 14(1):147-152.

24. Foucault M. O nascimento da medicina social. Microfísica do Poder. São Paulo: Edições Graal; 2010.

25. Marquetti FC. O Suicídio como Espetáculo na Metrópole: Cenas, Cenários e Espectadores. São Paulo: Editora Fap-Unifesp; 2011.

26. Instituto de Pesquisa Econômica Aplicada (IPEA). Fórum Brasileiro de Segurança Pública. Atlas da violência 2019 [Internet]. Brasília, Rio de Janeiro, São Paulo: IPEA; 2019. [acessado 2020 fev 04]. Disponível em: http://www.ipea.gov.br/atlasviolencia/download/12/ atlas-2019.

27. Durkheim É. O suicídio: estudo de sociologia. São Paulo: Nacional; 1966.

28. Dias ML. Suicídio: Testemunhos de Adeus. São Paulo: Editora Brasiliense; 1991.

29. Pereira JS. História da pediatria no Brasil de final do século XIX a meados do século XX [tese]. Belo Horizonte: Universidade Federal de Minas Gerais; 2006.

30. Saraiva CB. Suicídio: de Durkheim a Shneidman, do determinismo social à dor psicológica individual. Psiquiatr Clinica 2010; 31(3):185-205.

Artigo apresentado em 06/03/2020

Aprovado em 31/03/2021

Versão final apresentada em 02/04/2021

Editores-chefes: Romeu Gomes, Antônio Augusto Moura da Silva 